\title{
EVALUACIÓN DEL APRENDIZAJE EN LA EDUCACIÓN FÍSICA ESCOLAR ${ }^{1}$
}

AVALIAÇÃO DA APRENDIZAGEM NA EDUCAÇÃO FÍSICA ESCOLAR

\author{
EVALUATION OF LEARNING IN SCHOOL PHYSICAL EDUCATION
}

\author{
Evandra Hein Mendes ${ }^{2}$ y leda Parra Barbosa Rinaldi ${ }^{3}$ \\ evandrahmendes@hotmail.com \\ parrarinaldi@hotmail.com \\ ${ }^{2}$ Universidad Estatal del Oeste de Paraná, Brasil ${ }^{3}$ Universidad Estatal de Maringá, \\ Brasil
}

Envío original: 2019-07-10 Reenviado: 2019-12-13 Aceptado: 2020-02-11

Publicado en versión en español: 2020-06-23

Doi: $\underline{\text { https://doi.org/10.15517/pensarmov.v18i1.42361 }}$

\begin{abstract}
Resumen
Este estudio analizó las características y los delineamientos del proceso de evaluación del aprendizaje en la Educación Física escolar. Para ello, se aplicó un cuestionario semiestructurado con preguntas abiertas sobre el proceso de evaluación de los estudiantes, a cuarenta y seis profesores de Educación Física de la red pública de enseñanza de Paraná. Los datos revelaron que los profesores evalúan las competencias cognoscitivas, físico-kinestésicas y la socioafectiva de los alumnos, utilizando como criterios de análisis la retención de información, la comprensión y aplicación de los conceptos, la evolución individual, el desempeño físico/técnico, la cooperación, la participación y el comportamiento de los alumnos, por medio de pruebas teóricas, trabajos y observaciones.
\end{abstract}

Palabras clave: evaluación, práctica pedagógica, educación física.

\section{Resumo}

Esse estudo analisou as características e delineamentos do processo de avaliação da aprendizagem na educação física escolar. Para tanto, foi aplicado um questionário semi-

\footnotetext{
${ }^{1}$ Artículo traducido al español: Versión original en portugués disponible en: Hein Mendes, E., \& Barbosa Rinaldi, I. (2020). Avaliação da aprendizagem na Educação Física Escolar. Pensar en Movimiento: Revista de Ciencias del Ejercicio y la Salud, 18(1), e38295. doi: https://doi.org/10.15517/pensarmov.v18i1.38295
} 
estruturado com questões abertas sobre o processo de avaliação dos estudantes a quarenta e seis professores de educação física da rede pública de ensino do Paraná. Os dados revelaram que, os professores avaliam as competências cognitiva, fisicocinestésica e a socioafetiva dos alunos, utilizando como critérios de análise a retenção de informações, a compreensão e aplicação dos conceitos, a evolução individual, o desempenho físico/técnico, a cooperação, a participação e o comportamento dos alunos, por meio de provas teóricas, trabalhos e observações.

Palavras-chave: avaliação, prática pedagógica, educação física

\begin{abstract}
This study analyzed the characteristics and outlines of the evaluation process of learning in school physical education. Therefore, a semi-structured questionnaire with open questions about the process of student evaluation was applied to forty-six physical education teachers of the public school system of Paraná. The data revealed that the teachers evaluated the cognitive, physical-kinesthetic and socio-affective skills of the students, using as criteria of analysis the retention of information, the understanding and application of concepts, individual evolution, physical / technical performance, cooperation , the participation and the behavior of the students, through theoretical tests, works and observations.
\end{abstract}

Keywords: evaluation, pedagogical practice, physical education

\title{
Introducción
}

El abandono escolar precoz y los altos índices de reprobación de los estudiantes, han generado en el actual panorama educativo brasileño, innumerables discusiones sobre la función de la evaluación escolar y el papel de la escuela (Araújo, 2015). De manera general, el rumbo de la educación en el país es trazada en los documentos oficiales como la Ley de Directrices y Bases de la Educación (LDB, por sus siglas en portugués) n 9394/96, los Parámetros Curriculares Nacionales (PCN's), las Directrices Curriculares Nacionales (DCN's) y la Base Nacional Común Curricular (BNCC), que han indicado la creciente necesidad de que la escuela asuma un papel más social y contribuya a la formación humana, basada en la educación crítica. 
Con respecto a la evaluación escolar, los documentos que orientan la educación, destacan la necesidad de utilizar procedimientos de evaluación formativa que tomen en cuenta los contextos y las condiciones de aprendizaje, observando las trayectorias individuales o colectivas para identificar logros, avances, dificultades y posibilidades de aprendizaje (Brasil. Ministério da Educação, 2018).

Por lo tanto, es imprescindible modificar las practicas evaluativas utilizadas estrictamente para medir y cuantificar la capacidad del alumno de memorizar o reproducir conocimiento al final de una unidad didáctica. Además, no puede ser dirigida a la selección, promoción o clasificación de los alumnos (Souza, 2005; Calderón y Borges, 2013).

Por el contrario, la evaluación debe ser comprendida como un elemento integrante y regulador del proceso de enseñanza-aprendizaje, en el que la intención del profesor sea reunir elementos para estructurar situaciones que contribuyan con la evolución de los alumnos en diferentes aspectos. Desde esta perspectiva, el profesor y los alumnos podrán tomar decisiones asertivas sobre los rumbos de la educación (Brasil. Conselho Nacional de Educação, 2002; Palafox y Terra, 1998; Rios y Alsina, 2002).

Incorporar e implementar estrategias formativas para evaluar el aprendizaje, exige de los profesores un ejercicio de repensar conceptos y paradigmas ya establecidos en el área educacional, en especial en la Educación Física, en la que históricamente el proceso de evaluación estuvo vinculado a la cuantificación y a la medición de los comportamientos observables, como el desempeño físico, motor o técnico, con criterios establecidos en la lógica del rendimiento deportivo. Las principales consecuencias generadas con la utilización de ese modelo evaluativo fueron una clasificación y exclusión de los alumnos que no encajaban en la categoría de los más habilidosos o aptos físicamente, vinculándose con experiencias negativas con la Educación Física y generando un sentimiento de incapacidad y desvalorización (Darido, 1999).

A pesar de esta herencia histórica, en los días actuales, estudios sobre la evaluación en la Educación Física escolar (Mendes, 2007; Fernandes y Grenville, 2007; Santos y Maximiniano, 2013) han señalado que la evaluación ha sido menos enfocada en la cuantificación y al producto. Hay indicios de que la comunidad escolar ha reconocido la necesidad de implementar un carácter formativo en la evaluación escolar, ya que en algunos contextos los profesores han privilegiado la evaluación cualitativa, que se enfatiza en el proceso, con el objetivo de incluir todos los alumnos en las clases y mejorar su interés y su motivación para la ejecución de las actividades propuestas.

Sin embargo, se sabe que, en el contexto educativo, los cambios se establecen lentamente, ya que implican un redimensionamiento de paradigmas de pensamientos, 
o repensar la enseñanza en sí misma y los criterios utilizados para analizar el aprendizaje, hasta entonces, en el caso de la Educación Física, regidos por tecnicismos, por los errores o aciertos y por la búsqueda de alcanzar excelencia técnica y física (Darido, 1999).

En vista de este panorama de reflexiones y posibles cambios pedagógicos, surge el interés de investigar en el contexto educativo actual de la Educación Física escolar, las características y los delineamientos del proceso de evaluación del aprendizaje, identificando los objetivos y las funciones que los profesores atribuyen a la evaluación, a las competencias o comportamientos evaluados, a los criterios de análisis y a las estrategias o procedimientos utilizados.

\section{Metodología \\ Participantes}

Participaron en la investigación 46 profesores de Educación Física (tabla 1), que laboraban en escuelas públicas del Estado de Paraná, tanto en enseñanza fundamental como en enseñanza Media. La mayor parte de la muestra fueron mujeres, con edad entre 41 a 50 años y título de especialista. Respecto al tiempo como docentes, se percibió que el grupo estuvo constituido de forma equilibrada por profesores que laboraron en la profesión hasta 19 años, y por profesores con ejercicio profesional de 20 años o más, pertenecientes a los últimos ciclos de la carrera docente.

Además de trabajar en las escuelas, los profesores de Educación Física, cursaban el Programa de Desarrollo Educativo (PDE, por sus siglas en portugués) ofrecido por la Secretaría de Educación del Estado de Paraná. EI PDE es un programa de formación permanente, que permite a los profesores progresar en la carrera docente y mejorar la práctica pedagógica, con la participación en cursos desarrollados en universidades en Paraná y la realización de investigación desarrollada en la escuela donde laboran. La inserción en la Educación Física escolar y la experiencia pedagógica de los profesores, en conjunto con las discusiones teórico-metodológicas desarrolladas durante su participación en el programa, permiten la recopilación de datos relevantes, que pueden colaborar en la investigación de los elementos constitutivos de la evaluación en esta disciplina escolar. 
Tabla 1

Características de los profesores investigados

\begin{tabular}{|c|c|c|c|}
\hline Características & Ítems & $f$ & $\%$ \\
\hline \multirow{3}{*}{ Edad } & 30 a 40 & 16 & 35 \\
\hline & 41 a 50 & 26 & 57 \\
\hline & 51 a 60 & 4 & 8 \\
\hline \multirow{2}{*}{ Sexo } & Femenino & 30 & 65 \\
\hline & Masculino & 16 & 35 \\
\hline \multirow{2}{*}{ Tiempo de docencia } & hasta 19 años & 23 & 50 \\
\hline & 20 años o más & 23 & 50 \\
\hline \multirow{4}{*}{$\begin{array}{l}\text { Nivel en el que } \\
\text { laboran }\end{array}$} & $\begin{array}{l}\text { Enseñanza Fundamental } \\
\text { (Primaria) }\end{array}$ & & \\
\hline & Enseñanza Media & 11 & 24 \\
\hline & (Secundaria) & 5 & 11 \\
\hline & $\begin{array}{l}\text { Enseñanza Fundamental } \\
\text { y Media (Primaria y } \\
\text { Secundaria) }\end{array}$ & 30 & 65 \\
\hline \multirow{2}{*}{ Escolaridad } & Graduación & 2 & 4 \\
\hline & Especialización & 44 & 96 \\
\hline
\end{tabular}

Fuente: elaboración propia

\section{Instrumentos de recolección de datos}

Para obtener los datos, se utilizó un cuestionario con preguntas abiertas, del cual se obtuvo la validez de contenido (Bardin, 2009), a partir de tres dimensiones: claridad del lenguaje (comprensión de los términos y las expresiones textuales), la pertinencia práctica (grado de importancia de los ítems para el tema abordado) y la relevancia teórica (relación con los supuestos teóricos del área). Para ello, un grupo de evaluadores analizó cada indicador y determinó la pertinencia atribuyendo un concepto que correspondía a "1 - inadecuando, 2 - poco adecuado, 3 - aceptable, 4 - adecuado y 5 - muy adecuado". Además, los evaluadores tuvieron la posibilidad de expresar sus opiniones o sugerencias en los espacios colocados debajo de cada ítem, indicando modificaciones que consideraran necesarias, especialmente en los ítems que presentaran puntuaciones de inadecuado o poco adecuado.

Para la selección de los evaluadores del cuestionario, se determinó que debían cumplir al menos dos criterios de los cinco establecidos, a saber: poseer título de máster o doctor en Educación Física, ser alumno de doctorado en Educación Física, ser 
docente universitario preferiblemente en la carrera de Educación Física y de las subáreas socio cultural y pedagógica, profesor de enseñanza fundamental o media, ser miembro de un grupo de investigación vinculado a los cursos de graduación (bachillerato o licenciatura) en Educación Física.

Los resultados fueron catalogados y analizados por medio del porcentaje de concordancia inter-observadores, considerándose un ítem válido al presentar índice de concordancia mayor o igual a $80 \%$. Al observar los datos de cada ítem, se encontró que la claridad del lenguaje presentó $83 \%$ de concordancia, la relevancia teórica $98 \%$ y la pertinencia práctica $98 \%$, totalizando un puntaje de concordancia general de $93 \%$, porcentajes de concordancia considerados satisfactorios.

\section{Procedimientos de recolección de datos}

Posterior a la validación del instrumento, se realizaron contactos por medio de cartas de presentación para solicitar la autorización para la investigación. Teniendo las autorizaciones respectivas, se inició la recolección de los datos en las instalaciones de las instituciones que ofrecen el PDE en la región norte, noreste, oeste y suroeste de Paraná, en periodos de clases/cursos con fecha previamente agendada por la investigadora con el coordinador local del programa. Los participantes de la investigación recibieron información sobre los objetivos del estudio y firmaron la fórmula de Consentimiento Informado (TCLE, por sus siglas en portugués) para declarar su anuencia a participar en el estudio. El proyecto de investigación fue presentado al Comité de Ética en Investigación (COPEP, por sus siglas en portugués) con Seres Humanos de la Universidad de Maringá y aprobado en la resolución número 1.329.180.

Análisis estadístico

Para el análisis de los datos obtenidos en esta investigación, se utilizó estadística descriptiva calculando frecuencias y porcentajes. Además, se realizó la técnica de análisis de contenido, que incluyó un pre-análisis, una exploración de material y análisis de los resultados (Cassep-Borges, Balbinot y Teodoro, 2010).

\section{Resultados}

Los profesores investigados atribuían a la evaluación del aprendizaje en la Educación Física varios propósitos y funciones (tabla 2), relacionados tanto con una perspectiva cuantitativa, en la cual la intención era verificar la retención de los conocimientos, como la perspectiva formativa, en la que el foco de análisis era la evolución de los alumnos y evaluar la efectividad de la enseñanza y de la actuación 
profesional. Además de los propósitos relacionados con el proceso de enseñanza y aprendizaje, los profesores indicaron otros propósitos para la evaluación de los alumnos, como cumplir una exigencia burocrática y darle valor a la disciplina en el ambiente escolar.

\section{Tabla 2}

Propósitos y funciones de la evaluación en la Educación Física Escolar

\begin{tabular}{lcc}
\hline Propósitos y funciones & $f$ & $\%$ \\
\hline Verificar la retención del conocimiento & 30 & 42 \\
Analizar la evolución de los alumnos & 15 & 21 \\
Evaluar la efectividad de la enseñanza y la actuación & 14 & 20 \\
profesional & 10 & 14 \\
Cumplir una exigencia burocrática & 1 & 1 \\
Promover la participación del alumno & 1 & 1 \\
Valorizar la disciplina en el ambiente escolar & 71 & 100 \\
\hline Total
\end{tabular}

Fuente: elaboración propia

En el transcurso del proceso de evaluación los profesores utilizaban diferentes criterios de análisis para las competencias físico-kinestésicas, socioemocional, y cognitiva (tabla 3). En la competencia físico-kinestésica, el criterio más utilizado fue la evolución técnica y motora, en la competencia socioemocional, la socialización y la cooperación y en la competencia cognitiva, la retención del conocimiento. 


\section{Tabla 3}

Competencias evaluadas y criterios utilizados

\begin{tabular}{|c|c|c|c|}
\hline Competencias & Criterios de análisis & $f$ & $\%$ \\
\hline \multirow{4}{*}{$\begin{array}{c}\text { Físico/ } \\
\text { Kinestésica }\end{array}$} & Evolución técnica y motora & 36 & 22 \\
\hline & Desempeño en el juego/actividades & 7 & 4 \\
\hline & Dominio motor & 3 & 2 \\
\hline & $\begin{array}{l}\text { Comprensión del movimiento y de las } \\
\text { actividades }\end{array}$ & 3 & 2 \\
\hline \multirow{4}{*}{ Cognitiva } & Retención de información & 20 & 12 \\
\hline & Compresión de los contenidos & 14 & 8 \\
\hline & $\begin{array}{l}\text { Incorporación y aplicación de los } \\
\text { conceptos }\end{array}$ & 6 & 4 \\
\hline & Capacidad de argumentación & 3 & 2 \\
\hline \multirow{6}{*}{ Socioemocional } & Socialización y cooperación & 30 & 18 \\
\hline & $\begin{array}{l}\text { Participación efectiva y participación en } \\
\text { clase }\end{array}$ & 18 & 10 \\
\hline & Respeto a las reglas y al espíritu colectivo & 16 & 10 \\
\hline & Comportamiento y respeto al profesor & 8 & 5 \\
\hline & Actitudes hacia las diferencias individuales & 3 & 2 \\
\hline & Total & 167 & 100 \\
\hline
\end{tabular}

Fuente: elaboración propia

Algunos de los criterios utilizados por los profesores estaban vinculados al abordaje cuantitativo, como el desempeño y dominio motor y la retención de información. Por el contrario, la mayor parte de los criterios de análisis estaban vinculados con el abordaje cualitativo, como la percepción de la evolución del alumno, la comprensión de las actividades y de los contenidos, la incorporación y aplicación de los conceptos, la capacidad de argumentación, la socialización y la cooperación, la participación efectiva y la participación en el aula, el respeto a las reglas y el espíritu colectivo, el comportamiento, el respeto al profesor y a las actitudes hacia las diferencias individuales.

Para evaluar el aprendizaje de los alumnos en la Educación Física, los profesores utilizaban diversas estrategias, entre ellas la observación, las pruebas teóricas, las pruebas orales, los trabajos/seminarios/investigaciones y la autoevaluación (tabla 4). 
Tabla 4

Estrategias de evaluación utilizadas por profesores de Educación Física

\begin{tabular}{lcc}
\hline Estrategias/procedimientos & $\mathrm{f}$ & $\%$ \\
\hline Observación & 32 & 30 \\
Prueba Teórica & 29 & 26 \\
Trabajos/Seminario/Investigación & 26 & 24 \\
Prueba Práctica & 16 & 15 \\
Autoevaluación & 4 & 4 \\
Prueba Oral & 1 & 1 \\
\hline Total & 108 & 100 \\
\hline
\end{tabular}

Fuente: elaboración propia

\section{Discusión}

En la actualidad, las principales discusiones pedagógicas sobre la evaluación señalan la necesidad de modificar el carácter clasificatorio y punitivo adoptado a lo largo del tiempo a partir de la utilización del enfoque cuantitativo del proceso de evaluación, por un enfoque formativo, traspasando la espera del alumno y la emisión de conceptos o notas (Araújo, 2015). La implementación del enfoque formativo en la evaluación implica identificar progresos y resistencias en el aprendizaje por medio de reflexiones sobre la acción, para obtener datos que posibiliten orientar la planificación y regular la enseñanza, lo que puede, incluso, colaborar con la formación del sentido crítico del alumno (Brasil. Conselho Nacional de Educação, 2002).

La concepción de medida (Melo, Ferraz y Nista-Piccolo, 2010; Santos y Neto, 2003; Soares, 1992; Ramalho, Almeida, Machado, Santos y Nobre, 2012) y la evaluación como obligación o exigencia burocrática (Silva, 1999; Souza, 1993; Bratisfische, 2003) todavía impregnan la práctica pedagógica de la Educación Física escolar. Por el contrario, el sentido de la evaluación puede ser ampliado y tornarse en un espacio de registro e interpretación de datos, basados en el ejercicio constante de lectura de señales y de indicios de la evolución y del aprendizaje (Santos y Gonçalves, 1996; Amaral y Diniz, 2009; Ramiro, 2011; Zabala, 1999). Para esto, es necesario, a priori, comprender al estudiante como un ser en desarrollo y construcción permanente (Luckesi, 2010) y a posteriori, modificar el papel de la evaluación como un instrumento 
que ejerce presión y que sirve apenas para atribuir un concepto, por algo útil a profesores, estudiantes y la escuela (Santos, 2008; Darido, 2012; Betti y Zuliani, 2002).

El vínculo de la Educación Física con el movimiento humano y la relación con la cultura corporal, genera algunas singularidades, que la diferencian, de las otras disciplinas escolares más vinculadas a conocimientos de literatura y de escritura (Santos, Mathias, Matos y Vieira, 2015). El énfasis de la enseñanza y, en consecuencia, de la evaluación, recae en las competencias físico-kinestésicas y socioemocionales (Ramalho et al., 2012), en las que se valoriza la dimensión del saber hacer y las vivencias prácticas de los contenidos.

Sin embargo, la práctica requiere ser acompañada de la reflexión teórica para tener un significado de praxis, relacionando lo que fue aprendido a los conocimientos y a las experiencias personales ya incorporadas en la vida cotidiana (Darido, 2012). Además, estas particularidades no excluyen la posibilidad de materializar el conocimiento incorporado en dispositivos teóricos, como frases, textos y dibujos, para posibilitar que el alumno se exprese por medio de diferentes lenguajes, entre ellos el corporal, la escritura y el habla (Darido, 1999).

Elaborar un proceso de evaluación exige al profesor tomar decisiones acerca de los aspectos a ser evaluados, de los criterios de análisis y de las estrategias o procedimientos a ser utilizados para recolectar los datos que indiquen el aprendizaje de los alumnos (Ramiro, 2011). En la Educación Física, las estrategias más comúnmente utilizadas son la observación, las pruebas teóricas y los trabajos/seminarios/investigaciones, además de estas, hay un registro de uso de evaluaciones prácticas (Ramalho et al., 2012), autoevaluación (Bratisfische, 2003) y de producción de informes (Fernandes y Grenville, 2007).

En el contexto de la Educación Física, la observación ha sido ampliamente utilizada por los profesores para evaluar el aprendizaje del movimiento (Palafox y Terra, 1998). De manera general, la observación se presenta como una excelente fuente de información para conocer los avances del aprendizaje, cuando es construida con claridad de los objetivos de enseñanza y de los criterios de análisis, sin embargo, al convertirse en único criterio en la evaluación (Amaral y Diniz, 2009; Ramiro, 2011), sin cualquier intencionalidad o producción durante las actividades, puede generar falta de compromiso de los alumnos con la disciplina y desvincularse del proceso de enseñanza.

A pesar del carácter práctico de los contenidos de esta disciplina, imponer la necesidad de que el alumno vivencie las enseñanzas para aprenderlas, vincula la evaluación únicamente a la participación en clase empobreciendo el proceso. En lugar de aprovechar la evaluación del alumno para valorar la participación en las actividades, esta puede ser estimulada por medio de metodologías que faciliten el pensamiento 
divergente, la creación y las situaciones-problemas, para que la evaluación no sea únicamente de la presencialidad, sino también de descubrir diferentes formas de aprender y pensar sobre la práctica.

De esta forma, los trabajos, seminarios y las investigaciones son importantes herramientas para mejorar y enriquecer el aprendizaje sobre determinado tema; sin embargo, su enfoque debe ir más allá del sentido limitado a la formalización de una calificación, constituyéndose en una posibilidad de aprendizaje y de feedback para el profesor. Además, la reflexión conceptual de los contenidos de esta disciplina no puede descuidarse (Darido, 1999).

Las pruebas teóricas, en general, menos utilizadas en el área, han sido incorporadas en la actualidad en el proceso de evaluación para atender las normativas establecidas por las escuelas, que registran en el proyecto político pedagógico el sistema de evaluación a ser aplicado por todas las disciplinas, inclusive la Educación Física. Las pruebas prácticas, ampliamente utilizadas en el pasado para evaluar el desempeño físico y técnico de los alumnos, se presentan como estrategias menos utilizadas por los profesores para evaluar a los alumnos.

\section{Conclusiones}

La realización de este estudio permitió conocer de una mejor manera el proceso de evaluación del aprendizaje aplicado por profesores de Educación Física en la enseñanza Fundamental y Media del estado de Paraná, específicamente sobre las funciones atribuidas a la evaluación, a los criterios utilizados para analizar el aprendizaje de los alumnos en diferentes competencias y las estrategias utilizadas para evaluar.

$\mathrm{Al}$ analizar las funciones atribuidas a la evaluación se verificó que los profesores pretendían tanto, cuantificar la retención de los conocimientos de los alumnos y atender una exigencia burocrática, como analizar la evolución de los alumnos, reflexionando sobre la efectividad de la enseñanza y de su actuación profesional.

De la misma forma, al elaborar los criterios de análisis del aprendizaje los profesores revelaron su preocupación en evaluar las competencias cognitiva, físicokinestésica y socioafectiva de los alumnos, buscando cuantificar tanto la retención de conocimientos, el desempeño físico/técnico, el dominio motor de los estudiantes, el comportamiento y las actitudes de los alumnos durante la clase, como analizar la comprensión y aplicación de los conceptos relacionados a los contenidos de cultura corporal, la evolución individual, la socialización, la cooperación, la participación y el compromiso del alumno en el proceso de enseñanza y aprendizaje. 
Por último, al analizar las estrategias utilizadas por los profesores de Educación Física para evaluar, se pudo observar una preocupación en recolectar datos diferenciados que pudieran indicar diversos aspectos relacionados con el aprendizaje de los alumnos. De esta forma, para apoyar la construcción del concepto o calificación, los profesores utilizaban instrumentos evaluativos, basados tanto en la objetividad como en la subjetividad, tales como pruebas orales, teóricas y prácticas, observaciones, trabajos, seminarios y autoevaluación.

De manera general, se concluye que, en el contexto investigado, dos modelos evaluativos distintos comparten espacio y se evidencian en la práctica pedagógica de la Educación Física, ilustrando que los profesores utilizan tanto el enfoque cuantitativo, basado en la objetividad y la medición, como el enfoque cualitativo de carácter formativo y con énfasis en la subjetividad.

Considerando que este estudio analizó las prácticas evaluativas desarrolladas únicamente por profesores de Educación Física que laboraban en la enseñanza pública del estado de Paraná, se sugiere la ejecución de otros estudios, que involucren instituciones privadas de enseñanza para ampliar el alcance del contexto de análisis, así como la participación de la enseñanza fundamental en los primeros años y en la educación infantil. 


\section{Referencias}

Amaral, S.C.F. y Diniz, J. (2009). A avaliação na educação física escolar: uma comparação entre as escolas tradicional e ciclada. Movimento: Revista de Educação Física da UFRGS, 15(1), 241-258. Recuperado de https://seer.ufrgs.br/Movimento/article/view/2085

De Araújo, F.M.R. (2015). A avaliação formativa e seu impacto na melhoria da aprendizagem (Tese Doutorado em Educação Física). Universidade de Lisboa, Lisboa. Recuperado de http://hdl.handle.net/10400.5/8344

Bardin. L. (2009). Análise de Conteúdo. Lisboa, Portugal: Edições 70.

Betti, M. y Zuliani, L.R. (2002). Educação física escolar: uma proposta de diretrizes pedagógicas. Revista Mackenzie de Educação Física e Esporte, 1(1), 73-81. Recuperado de http://editorarevistas.mackenzie.br/index.php/remef/article/view/1363/1065

Brasil. Conselho Nacional de Educação. (2002). Resolução CNE/CP, de 18 de fevereiro de 2002. Brasília: Ministério da Educação. Recuperado de http://portal.mec.gov.br/cne/arquivos/pdf/rcp01 02.pdf

Brasil. Ministério da Educação. (2018). Base Nacional Comum Curricular: Educação é a Base. Brasil: Ministério da Educação; Governo Federal do Brasil. Recuperado de http://basenacionalcomum.mec.gov.br/

Bratisfische, S.A. (2003). Avaliação em Educação Física: um desafio. Journal of Physical Education, 14(2), 21-31. Recuperado de http://www.periodicos.uem.br/ojs/index.php/RevEducFis/article/view/3466

Calderón, A.I. y Borges, R.M. (2013). Avaliação Educacional: Uma abordagem à luz das revistas científicas brasileiras. Revista Iberoamericana de Evaluación Educativa, 6(1), 167-183. Recuperado de https://revistas.uam.es/index.php/riee/article/view/3849

Cassep-Borges, V., Balbinotti, M.A.A. y Teodoro, M.L.M. (2010). Tradução e validação de conteúdo: uma proposta para adaptação de instrumentos. In: L. Pasquali (Org.), Instrumentação psicológica: fundamentos e prática (pp.506-520). Porto Alegre: Artmed.

Darido, S.C.A. (1999). A avaliação em educação física escolar: das abordagens à prática pedagógica. In: Seminário de Educação Física Escolar (pp.50-66). São Paulo: Escola de Educação Física e Esportes da Universidade de São Paulo. Recuperado de https://www.scribd.com/document/266815518/A-Avaliacao-Em-Educacao-FisicaEscolar-Das-Abordagens-a-Pratica-Pedagogica-Anais-Usp-1999 
Darido, S. C. (2012). Educação física e temas transversais na escola. Campinas, SP: Papirus.

Fernandes, S. y Greenvile, R. (2007). Avaliação da aprendizagem na educação física escolar. Motrivivência: Revista de Educação Física, Esporte e Lazer, 19(28), 120138.

Recuperado de https://periodicos.ufsc.br/index.php/motrivivencia/article/view/6473

Luckesi, C. (2010). Avaliação da aprendizagem escolar (20 ed.). São Paulo: Cortez

Mendes, E.H., Do Nascimento, J.V. y Mendes, J.C. (2007). Metamorfoses na avaliação em Educação Física: da formação inicial à prática pedagógica escolar. Movimento, 13(2), 55-76.

Recuperado de https://seer.ufrgs.br/Movimento/article/download/3546/1947

De Melo, L.F., Ferraz, O.L. y Nista-Piccolo, V.L. (2010). O portfólio como possibilidade de avaliação na educação física escolar. Revista da Educação Física/ UEM, 21(1), 87-97. doi: https://doi.org/10.4025/reveducfis.v21i1.7090

Palafox, G.H.M. y Terra, D.V. (1998). Introdução à avaliação na Educação Física escolar. Pensar a Prática, 1(1), 23-37.

Da Ramalho, M.H.S., de Almeida, C.R., Machado, Z., dos Santos, J.O.L. y Nobre, G.C. (2012). Avaliação na educação física escolar: uma análise a partir do modelo de inteligência motora. Pensar a prática, 15(4), 821-1113. doi: https://doi.org/10.5216/rpp.v15i3.14528

Rios, O.B. y Alsina, I.B. (2002). Estratégias de avaliação. In: S. La torre y O. B. Rios (orgs.). Curso de formação para educadores: estratégias didáticas inovadoras. São Paulo: Madras.

Dos Santos, S.G. y Gonçalves, G. (1996). Avaliação em Educação Física: uma análise nas escolas estaduais e municipais da cidade de Maringá-PR. Revista da Educação Física/EUM, 7(1), 75-83. Recuperado de https://pdfs.semanticscholar.org/17dd/5770dd57564e90573565cc57e93962128aa4. $\underline{\mathrm{pdf}}$

Santos, W. (2008). Currículo e avaliação na Educação Física: práticas e saberes. In: O. Schneide, J.T. Grunnenvaldt, R. Khun y S.D.D. Ribeiro (Orgs.). Educação Física esporte e sociedade: temas emergentes, 2, 87-106.

Dos Santos, W. y Maximiano, F. L. (2013). Avaliação na educação física escolar: singularidades e diferenciações de um componente curricular. Revista Brasileira de Ciências do Esporte, 35(4), 883-896. doi: https://doi.org/10.1590/S0101$\underline{32892013000400006}$

Dos Santos, W., Mathias, B.L.., Matos, J.M.C. y Vieira, A.O. (2015). Avaliação na educação física escolar: reconhecendo a especificidade de um componente 
curricular. Movimento: Revista de Educação Física da UFRGS, 21(1), 205-218. doi: https://doi.org/10.22456/1982-8918.46895

Santos, W. y Neto, A.F. (2003). Avaliação na educação física escolar: o debate acadêmico em periódicos. In: Encontro fluminense de educação física escolar, 7. Rio de Janeiro. Recuperado de www.proteoria.org

Da Silva, A.H. (1999). A avaliação da aprendizagem em educação física escolar: desvelando a categoria. Pensar a Prática, 2(1), 101-118.

Da Silva Ramiro, F. (2011). A avaliação da aprendizagem na educação física escolar em Ferraz de Vasconcelos (Dissertação mestrado). Universidade São Judas Tadeu, São Paulo.

Soares, C.L., Castellani Filho, L., Taffarel, C.N.Z., Varjal, E., Escobar, M.O. y Bracht, V. (1992). Metodologia do ensino da Educação Física. São Paulo: Cortez

Souza, C.P. (2005). Preconceito, discriminação e exclusão nas aulas de educação física na visão dos alunos (Trabalho de Conclusão de Curso Graduação em Educação Física). Instituto de Biociências, Universidade Estadual Paulista, Rio Claro.

Souza, N.P. (1993). Avaliação na Educação Física. In: S.Votre (org). Ensino e avaliação em Educação Física. São Paulo: Ibrasa

Zabala, A. (1999). A prática educativa: como ensinar. Porto Alegre: Artmed 